\title{
Investigation Report on the Inheritor of Provincial Intangible Cultural Heritage Sister Yue's Song in Shitang*
}

\author{
Qunying Wang \\ School of Music \\ Shaoguan University \\ Shaoguan, China
}

\begin{abstract}
Sister Yue's song is spread in Shitang ancient village of Renhua County, Shaoguan city in North Guangdong. It is selected as the third batch of provincial intangible cultural heritage of Guangdong Province on October 16, 2009. The singers of sister Yue's song are all women, so it is a kind of feminine folklore culture. The folk inheritance of sister Yue's song is the main channel to spread and inherit the folk music culture of sister Yue's song. Its heritage representatives are Tan Caixia, Li Zhaohuan, Li Yuqing and Dai Lianfeng. With the attention from multiple sides, the current inheritance situation of Yuejie song has contradictions, conflicts and truthfulness need to be solved because of the change of its ecological environment.
\end{abstract}

Keywords-sister Yue's song; inheritor; inheritance situation; ecological environment

\section{INTRODUCTION}

Sister Yue's song is spread in Shitang ancient village of Renhua County, Shaoguan city in North Guangdong. It is selected as the third batch of provincial intangible cultural heritage of Guangdong Province on October 16, 2009. Sister Yue's song originates from the Tang Dynasty (to be investigated), and its ceremonial activities are from August 1st to August 15th each year. The process is divided into three parts: "great sister Yue"-"fascinate sister Yue"-"send sister Yue". The singers of sister Yue's song are all women, so it is a kind of feminine folklore culture. During the activity of "Sister Yue's Hall", the female feelings and the pursuit of a better life are expressed through singing hundreds of songs that reflect the theme of daily life and local customs. Sister Yue's song is not only a sacrificial activity, but also a major part of the spiritual and cultural life of the local women.

The folk inheritance of sister Yue's song is the main channel to spread and inherit the folk music culture of sister

*Fund Project: Shaoguan City Social Science Planning 2016 (Shaoguan Culture Research Base) "Salvation Collection of inheritor of Provincial intangible cultural heritage Shitang sister Yue's song Heritage" (J2016012). 2017 Shaoguan University scientific research project

"Collection and Research of Music Activity Live of sister Yue's song in Shitang, Renhua" (SZ2017SK06)
Yue's song. The folk inheritance types of sister Yue's song are divided into two categories according to the difference in status of the spreader and the inheritor: group inheritance and individual inheritance activities. Group inheritance is a kind of natural inheritance mode formed in daily entertainment and folk custom activities. There is no so-called spreading and inheriting relationship between participating individuals, since each participant is both spreader and inheritor and unconscious learning is the established form of accepting inheritance. This way of inheritance is far-reaching. Individual inheritance can be divided into two categories with obvious relationship between teachers and apprentices and no obvious relationship between teachers and apprentices. The author have been following up on-the-spot research on Music Activities of sister Yue's song since 2012, and now an elaboration on the survey of its inheritors is made.

\section{INHERITANCE GENEALOGY OF SISTER YUE'S SONG}

According to legend, "sister Yue's song" was sung among mother-in-law, sister-in-law, and sisters in the way of oral teaching from the Tang Dynasty. Because of the age, the genealogy cannot be studied. Only after the establishment of the Progenitor spectrum in six years of Chongzhi in the Qing Dynasty did the following genealogy is established in "Table I" and "Table II": 
TABLE I. The Genealogy of Sister YuE's Song IN ShUANGFEng VILLAGE

\begin{tabular}{|c|c|c|c|c|c|}
\hline generation & Name & gender & date of birth & cultural level & Residence \\
\hline the first generation & Unknown & female & Qing dynasty & Unknown & Shitang village \\
\hline the second generation & Unknown & female & Qing dynasty & Unknown & Shitang village \\
\hline the third generation & Unknown & female & Qing dynasty & Unknown & Shitang village \\
\hline the fourth generation & Unknown & female & Qing dynasty & Unknown & Shitang village \\
\hline the fifth generation & Unknown & female & Qing dynasty & Unknown & Shitang village \\
\hline the sixth generation & Liu Suchun & female & 1908 & Unknown & Shitang village \\
\hline \multirow[t]{3}{*}{ the seventh generation } & Tan Caixia & female & 1923 & Unknown & Shitang village \\
\hline & Zhang Xibi & female & 1928 & illiteracy & Shitang village \\
\hline & Bian Meizai & female & 1928 & illiteracy & Shitang village \\
\hline \multirow[t]{3}{*}{ the eighth generation } & Li Dingmei & female & 1935 & primary school & Shitang village \\
\hline & Che Meihua & female & 1935 & primary school & Shitang village \\
\hline & Li Caiji & female & 1936 & primary school & Shitang village \\
\hline \multirow[t]{4}{*}{ the ninth generation } & Zhu Xinju & female & 1940 & junior high school & Shitang village \\
\hline & He Xinju & female & 1940 & junior high school & Shitang village \\
\hline & Huang Zhaoyun & female & 1943 & junior high school & Shitang village \\
\hline & Li shuijuan & female & 1948 & junior high school & Shitang village \\
\hline \multirow[t]{7}{*}{ the tenth generation } & Zeng Julian & female & 1950 & junior high school & Shitang village \\
\hline & Dai Furong & female & 1952 & junior high school & Shitang village \\
\hline & Zhang Cailian & female & 1953 & junior high school & Shitang village \\
\hline & Liu Jiarong & female & 1954 & junior high school & Shitang village \\
\hline & Peng Yinglan & female & 1955 & junior high school & Shitang village \\
\hline & Li Xiuzhao & female & 1955 & junior high school & Shitang village \\
\hline & Chen Meixiang & female & 1957 & junior high school & Shitang village \\
\hline \multirow[t]{8}{*}{ the eleventh generation } & He Qianying & female & 1962 & junior high school & Shitang village \\
\hline & Song Lianju & female & 1962 & junior high school & Shitang village \\
\hline & Xie Youcai & female & 1963 & junior high school & Shitang village \\
\hline & Dai Yifeng & female & 1964 & primary school & Shitang village \\
\hline & Dai Lianfeng & female & 1964 & primary school & Shitang village \\
\hline & Li Xiuqiu & female & 1964 & primary school & Shitang village \\
\hline & Li Changxiang & female & 1965 & primary school & Shitang village \\
\hline & Chen Huodi & female & 1965 & high school & Shitang village \\
\hline \multirow[t]{2}{*}{ the twelfth generation } & Li Simei & female & 1970 & junior high school & Shitang village \\
\hline & Zhu Yunhua & female & 1971 & junior high school & Shitang village \\
\hline the thirteenth generation & He Yanping & female & 1996 & primary school & Shitang village \\
\hline
\end{tabular}

TABLE II. The Genealogy of THE Sister YuE's Song IN Li's ANCESTRAL Temple OF New House

\begin{tabular}{|l|l|l|l|l|l|}
\hline \multicolumn{1}{|c|}{ generation } & \multicolumn{1}{c|}{ Name } & \multicolumn{1}{c|}{ gender } & \multicolumn{1}{c|}{ date of birth } & \multicolumn{1}{c|}{ cultural level } & \multicolumn{1}{c|}{ Residence } \\
\hline the first generation & Unknown & female & Qing dynasty & Unknown & Shitang village \\
\hline the second genertaion & Unknown & female & Qing dynasty & Unknown & Shitang village \\
\hline \multirow{5}{*}{ the third generation } & Lian Yunfeng & female & 1931 & primary school & Shitang village \\
\cline { 2 - 6 } & Tan Yizhao & female & 1934 & illiteracy & Shitang village \\
\cline { 2 - 6 } & Tan Zhengqing & female & 1936 & illiteracy & Shitang village \\
\hline \multirow{5}{*}{ the fourth generation } & Li Huizhen & female & 1946 & primary school & Shitang village \\
\cline { 2 - 6 } & Zeng Zhaozhao & female & 1948 & primary school & Shitang village \\
\hline \multirow{5}{*}{ the fifth generation } & Li Liu'e & female & 1954 & junior high school & Shitang village \\
\cline { 2 - 6 } & Zhang Jinju & female & 1955 & junior high school & Shitang village \\
\cline { 2 - 6 } & Li Baijuan & female & 1958 & junior high school & Shitang village \\
\hline & Li Yuqing & female & 1959 & primary school & Shitang village \\
\cline { 2 - 6 } & Li Yanfen & female & 1962 & high school & Shitang village \\
\cline { 2 - 6 } & He Haizhao & female & 1968 & junior high school & Shitang village \\
\cline { 2 - 6 } & Li Bixia & female & 1968 & junior high school & Shitang village \\
\hline the seventh generation & Mao Xinglian & female & 1972 & junior high school & Shitang village \\
\hline the eighth generation & Li Wenbo & female & 1994 & primary school & Shitang village \\
\cline { 2 - 5 } & Lian Caiping & female & 1998 & primary school & Shitang village \\
\cline { 2 - 5 } & He Xiaofang & female & 1998 & primary school & Shitang village \\
\cline { 2 - 5 } & Li Xianhui & female & 1998 & primary school & Shitang village \\
\cline { 2 - 5 } & Li Chunping & female & 1999 & Shimary school \\
\hline
\end{tabular}

b. This form is provided by intangible cultural heritage office of Cultural Center in Renhua County. Thanks for Director Lin Junjie.

It is said that there were eleven song halls in Shitang Village during the peak of sister Yue's song in Ming Dynasty. There are usually 30 to 40 members in each song hall. The members of each song hall are composed either due to family factors, congeniality, or neighborhood factors. On every Mid-Autumn Festival in August, members of the 
singing circle gather together to dispatch the Sister Yue's song circle through inviting the gods of the Sister Yue in the folklore to exchange emotions, express their feelings, and pin down the pursuit of a better life. Based on this, the inheritance genealogy is not complete and accurate. The Shuangfeng Village and Li's ancestral temple of the new house in the table are also cultural appellation generated under the concern of various sides such as intangible cultural heritage.

\section{FIELD INVESTIGATION OF INHERITORS OF INTANGIBLE Cultural Heritage Sister Yue's Song IN SHITANG}

Tan Caixia (1923-2017), born in Dongtang, Renhua County, and has a rich family. Tan Caixia is clever and studious since childhood. Influenced by her family, she knows melody and learns to sing sister Yue's song at the age of 6. She is betrothed when still in womb of mother to Li at 20. Tan Caixia is happy to teach the sisters of the ancient village the sister Yue's song, which reflects the production, life and emotion of women. In 1974, Tan Caixia assisted Li Zhaohuan, captain of the Literary Propaganda Team of the Battalion, in collecting materials and recordings of sister Yue's song for the Guangdong Music Research Office. In 1980, together with the Cultural Bureau of Renhua County, she collected the materials of sister Yue's song and compiled "four sets of integration" (stories, ballads, dances, and instrumental music). In 2004, she set up "sister Yue's song circle" in Shitang. In 2005, she organized "sister Yue's song" team to take part in various performances in Guangdong, Shaoguan and Renhua counties, including "Folk Culture Special Documentary Film" filmed by CCTV, "Travel through Shaoguan" filmed by Shaoguan TV Station and so on. In October 2007, Tan Caixia led the "sister Yue's song" team to take part in the provincial-level scientific and cultural activities jointly organized by Guangdong provincial cultural department, Guangdong provincial science and technology department, and Guangdong provincial committee of communist youth league, and won the honor of "the first Guangdong rural youth scientific and cultural activity month outstanding work" Since 2008, sister Yue's song has ushered in a golden period of development. Tan Caixia actively assisted the cultural department to do a good job in the collection and training of sister Yue's song, often instructed sister Yue's song Troupe to perform, and made a great contribution to the inheritance and development of sister Yue's song. In 2009, she assisted the culture department of the county and town successively to declare the sister Yue's song to be listed in the Intangible Cultural Heritage List of Shaoguan City, Guangdong Province. At the age of 86, she insisted on arranging and inheriting sister Yue's song in the form of oral teaching and recording by others and assisted the cultural department of Shitang town in arranging and composing standardizing lyrics and guiding performances of sister Yue's song. The works she participated in compiling and singing, such as "Season Song", "New Tea Collection" and "A Stalk", were widely circulated in Shitang ancient village. As a result, she has become the highest-rated and most prestigious person with the strongest organizing ability in sister Yue's song circle, and she also becomes the seventh generation of Yuejie's singing circles.
She is awarded the title of "Master of Folk Culture and Arts in Guangdong Province". Regrettably, Tan Caixia died in October 2017 at the age of 94 before completing the "Oral Recording History of inheritor of Provincial intangible cultural heritage" carried out by the Guangdong Provincial intangible cultural heritage Protection Center.

Li Zhaohuan, male, was born in Shitang Village, Renhua County in December 1951. As a folk artist, Li Zhaohuan has multiple identities and is engaged in teaching, organizing and writing all his life. In 1974, he led to establish the Shitang Battalion Literary Propaganda Team, and served as captain. In the autumn of 1975, he helped Zong Jiang, the director of the Guangdong Provincial Music Research Office, and Xian Defu, the director of the Renhua County Culture Bureau, to collect and sort out sister Yue's song for the first time. Li Zhaohuan was the guide and recorded the words. 17 sister Yue's songs are included in the Chinese Ballad Integration (Guangdong Volume) book. In 1980, Rao Jichou, Lai Zimin, and others from the Shaoguan Regional Culture Creation Room came to Shitang to collect information on sister Yue's song for the second time. Li Zhaohuan accompanied them all the time, 30 sister Yue's song were included in the book "Tea-picking Opera in Northern Guangdong." In 1982, the chapter "Hakka Folksongs" in "Chinese Folk Song Integration (Guangdong Volume)" complied by Zongjiang, Huang Zirong, Deng Jinyao included 17 sister Yue's song. In 1988 , integration editor of Shaoguan ballad again came to Shitang to collect sister Yue's song, and some lyrics were recorded in Shaoguan ballad integration book. In 2006, when Renhua County Government compiled the history of Renhua literature, Li Zhaohuan provided most of the lyrics of sister Yue's song in the book. There are "200 folk songs in northern Guangdong", "Renhua County Chronicle", and "Shitang Town Chronicle" and other books recording some content of sister Yue's song. In 2004, two stations of the south united shaoguan tv station went to Shitang to photograph Shitang sister Yue's song "all over Shaoguan". In July 2006, the CCTV-4 International Channel filming team went to Shitang to film "Looking for the Disappeared Shao Music"--Shitang sister Yue's song. At the end of 2009, Shitang sister Yue's song declared the provincial intangible cultural heritage successfully. In 2010, Renhua County's CPPCC went to Shitang Village to shoot the TV special column "Explore Shitang Village". Then Beijing TV Station photographs the Danxia Mountain scenery propaganda column, Guangdong Province place name record column, and Guangdong Province ancient post road contest propaganda column and so on. These large-scale publicity activities have pushed the Shitang sister Yue's song to the peak. In the attention of all levels of government, Renhua County TV station filmed "Li Zhaohuan sister Yue's song Feelings" special column for Li Zhaohuan. In addition to actively participating in the collection, sorting and dissemination of sister Yue's song, Li Zhaohuan also led the team to participate in the following performances; Danxia Xiafu Village in September 2010, Zhoutian Village in October 2010, Xia Fu Troupe in Shitang in 2011, Shaoguan Song and Dance Troupe in Shitang Tong for women's day, in November of the same year, the second China Hakka Culture 
Festival in Heyuan in Guangdong Province and win the Silver Award and so on.

Li Yuqing, female, with Han nationality, was born in Shitang town in August 1959. Growing up under the influence of "sister Yue's song" in Shitang, she began to learn sister Yue's song from the old people in the village when she was five or six years old. At that time, older people generally can sing sister Yue's song. Owing to the lack of recreation, the old people also sing sister Yue's song when they are doing farm work. Li Yuqing's grandmother, Tan Qiubi, was one of the singers who collected and sorted out sister Yue's song in early years. What was strange was that there was no name for her in the genealogy of application for the list of world heritage. "At the time of the Cultural Revolution, it was not allowed to sing sister Yue's song. Later on, some old people began to learn again," says $\mathrm{Li}$ Yuqing. In 2004, she joined the "Travel to Shaoguan" filming team organized by Master Li Zhaohuan. After that, she joined $\mathrm{Li}$ Zhaohuan in performing, publicizing and teaching and inheritance of the sister Yue's song circle of the $\mathrm{Li}$ ancestral temple in the new house, also known as the Yi De hall sister Yue's Team. Li Yuqing admits frankly, she has not done the detailed statistics and classification to the sister Yue's song. In March 2011, Li Yuqing was awarded by Shaoguan Municipal Bureau of Cultural Press and Publication as the representative inheritor of the municipal intangible cultural heritage project Shitang sister Yue's song.

Dai Lianfeng, female, with Han nationality, was born in Shitang town in February 1965. At the age of 11, she started singing with Tan Caixia. At that time, the Tai Lianfeng family lived in Triangle Street, Shitang Town. On the first day of August, 40 or 50 people gathered at Tan Caixia's house to sing sister Yue's song, until the end of the receiving sister Yue Ceremony on August 15. She felt that singing sister Yue's song is very comfortable and can make the mood good. The lyric of sister Yue's song is very interesting, and there are many positive teachings, so it is worth passing on. Dai lianfeng knows a few words. Since she started singing, she has consciously recorded the lyrics of sister Yue's song. She uses spelling or other homonyms to replace the words she does not know how to write, or she simply leaves them empty. The yellowish, shabby little book is painted with some mutilation Dai Lianfeng's sisters Dai Furong and Dai Yifeng are core members of the sister Yue team in Shuangfeng villige. She firmly believes that only she can give all the lyrics of sister Yue's songs, and only she can sing all the authentic sister Yue's songs. However, there is a deep contradiction between them and the sister Yue's song circle of the Li ancestral hall of the new house in the problem of striving for the inheritance of sister Yue's song. Dai Lianfeng participated in the sister Yue's song Show at the Spring Festival Gala in the county town in 2010, 2011 and 2012. In 2017 , she is awarded as the representative inheritor of the second batch of municipal intangible cultural heritage projects Shitang sister Yue's song by Shaoguan Municipal Bureau of Culture, Radio, Television and Press and Publication.

\section{The PRESENT SituAtion AND EXISTING PROBLEMS OF INHERITANCE OF SISTER YUE'S SONG}

In recent years, inheritors of sister Yue's song actively run sister Yue's song Training Course. The training course mainly fosters young people to perceive, inherit and learn sister Yue's song. There are now two inheritance bases of sister Yue's song. First is the new house Li ancestral temple sister Yue's song base with $\mathrm{Li}$ Yuqing and Li Zhaohuan responsible for the inheritance and singing. In the 1970s and 1980s, sister Yue's circle activity was held every year (August 1-August 15) in the Ancestral Hall of the Li family. In 2004, Li Zhaohuan set up a seven-person sister Yue's song Team. When teaching sister Yue's song, Li Zhaohuan first wrote down the lyrics and taught them sentence by sentence. After more than ten years of development, the Yide hall sister Yue team has nine teachers, divided into daily teaching and the summer and winter vacation children teaching. Children can apply voluntarily without charge. The age of students is about 5-12 years old, and there are 25 students attending formally in 2017. In the course, the members of sister Yue hall will take turns as teachers. They will teach the students to sing sister Yue's song, perform the dance of sister Yue's song and study the knowledge of history of sister Yue's song by oral teaching. Teaching time is 13:00--15:30 in the afternoon, and the teaching place in the Li ancestral hall for one month. So far, two stages of teaching have been completed. In addition to teaching inheritance, the students also actively participate in the art performance in Renhua County, and Shitang Town and so on.

The other is the teaching base of middle School in Shuangfeng billage in Shitang, which is headed by Dai Lianfeng. In 2017, Middle School of Shuangfeng Village in Shitang set up a Sister Yue team with 22 students. Some members participate voluntarily, some are selected by school teachers, and some are recommended by parents. The mode of teaching singing began when Dai Lianfeng went to the school for teaching and singing. Later, because the farm work was busy, and because the inheritors were strongly opposed to, now the school teacher go to Dai Lianfeng's home to record the sister Yue's song and teach them in class.

From this, it can be seen that the singing place, time, function and the overall background of faith of sister Yue's song have changed greatly, and the survival environment of Yuejie singing culture has been lost, so it will be inevitably difficult to maintain the genuineness of cultural inheritance.

\section{CONCLUSION}

Moon worship originates from the primitive celestial body worship. The moon is a feminine synonym, and the moon is "lunar", as a female symbol. In the Tang Dynasty, the custom of Mid-Autumn Festival and worship of the moon is extremely prosperous, and to the Song Dynasty, the worship activities in the court and the folk get much scale. Currently, there are still some historic sites such as the Moon Pavilion, the Moon Altar and the Moon Tower. After the Ming and Qing Dynasties, the activities of worship of the Moon are even grander. Shitang sister Yue's Song has been full of mysterious moon worship activities since its birth, 
development and circulation. Nowadays, sister Yue's Song, as a sign of Shitang tourism culture, has been changed from a solemn female sacrifice ceremony to a popular group form of relaxed entertainment. The protection of cultural ecology is an important guarantee for the sustainable development of traditional folk music activities. However, under the attention of intangible cultural heritage protection, the creation of folk festivals not only destroys the original timedomain functional culture, but also wastes a lot of money. The normal folk cultural life has become the economic product of exaggeration and appearance. Inheritors receive a monthly subsidy, but there is no self-esteem and conscious choice to train a good inheritance successor, and there is a trend of joining the commercial performance.

\section{REFERENCES}

[1] Wang Qunying. Exploration of Shitang sister Yue's song [J]. Journal of Shaoguan University, 2014 (3). 王群英. 石塘月姐歌探网[J].韶关 学院学院学报, 2014 (3).

[2] Jin Guangyi. The Production and Consumption of Ethnic Culture. Zhao Jiawen, Ma Rong, Editor-in-Chief, Ethnic Development and Social Change, Nationalities Publishing House, 2001 (7). 金光亿. 《民族文化的生产与消费》 赵嘉文、马戎主编《民族发展与社 会变迁》，民族出版社，2001(7).

[3] Wang Qunying. Artistic Features of "sister Yue's song" in Shitang Village, Renhua County in North Guangdong [J]. Journal of Xinghai Conservatory of Music, 2014 (3). 王群英. 粤北仁化县石塘古村 “月 姐歌" 的艺术特征 [J].星海音乐学院学报, 2014（3）. 